\title{
SOFTWARE CATALOGUE FOR SCIENTIFIC CONTEXT EXPLICATION
}

\author{
Olga Kononova ${ }^{1}$, Dmitry Prokudin ${ }^{1,2}$ and Angelina Timofeeva ${ }^{1}$ \\ ${ }^{1}$ ITMO University 197101, St. Petersburg, Russia \\ ${ }^{2}$ St. Petersburg State University 199034, St. Petersburg, Russia
}

\begin{abstract}
Computerization of scientific activity has changed the interdisciplinary study mechanics and researchers' views, providing new opportunities for analysis. This paper presents a catalogue of analytical software widely used for contextual knowledge explication and scientific text mining. The catalogue focuses on the contextual knowledge types (contexts) processed with a software considered, and applies both, human-oriented and machine-oriented IS descriptions. The human-oriented description of a software catalogue is presented by a corresponding IS architecture (applications and data) on conceptualization level. The machine-oriented software description is performed with Dublin Core metadata. The Dublin Core metadata have allowed structuring the main software characteristics and provided the metadata export to other presentation formats to be place in various kinds of ISs and metadata aggregators. The machine-readable part of the catalogue performs the replenishment tasks, software effective search and its recognition in accordance with the research tasks. The practical implementation of the catalogue as an open access network platform by the OAI-PMH metadata exchange protocol provides the integration into the scientific information space as a researchers' guidance.
\end{abstract}

\section{KEYWORDS}

Human-Machine Interaction, Scientific Text Mining, Context, Software, Contextual Knowledge, IS Architecture, Architectural Description, Human-Machine Interaction, Dublin Core

\section{INTRODUCTION}

Modern multidisciplinary research makes extensive use of a software designed to explicate and analyze contextual knowledge. A variety of tools poses the challenge for researchers of multidisciplinary scientific fields to select a software most appropriate for their research objectives. The choice complexity lies in the lack of a software systematic description that can be used to solve a wide range of problems in scientific text mining, initially focused on business applications or solving problems in narrow subject areas (for example, linguistics). The paper describes the approach to software systematic presentation development for analysis and explication of contextual knowledge for research purposes based on identification of essential characteristics (classes of tasks to be performed, functions to be implemented and context types). Considering the existing software classifications, the main classes for contextual knowledge processing are identified and systematized. Based on the typology of contextual knowledge developed by the authors, types of contexts are distinguished as an essential software characteristics. The main functions implemented in the software are also allocated as a separate characteristics. The following is a structured description of a software to be presented in a catalogue form. Software systematization in the catalogue is performed on the enlarged classes of tasks. The catalogue is presented in two forms: human-readable and machine-readable. A human-readable catalogue representation proposes a software architecture at a conceptual level. The software architecture reflects the modules, functions, and types of contexts being processed.

The paper presents two cases to demonstrate the results obtained in the form of a structured description of T-Libla and Voyant-tools softwires. The developed systematized and structured presentation of the software replenishes the catalogue with new software, as well as ensures its effective selection for research purposes based on contextual knowledge explication and analysis. 


\section{SOFTWARE CLASSIFICATIONS AND TYPOLOGY OF CONTEXT SCIENTIFIC KNOWLEDGE}

The target users of the developed catalogue are scientists and teachers specializing in interdisciplinary research. They usually rely on the existing approaches to software classification. Both general approaches, that single out the most general software classes, and specific ones, that focus on a more detailed description of software subclasses applied in various fields, are developed. One of the general approaches to classify Business intelligence (BI) class software is Gartner's Infrastructure and Applications Worldwide Software Market Definitions, annually updated, which reflects Gartner's general approach to estimate software market development [Woodward A. et al, 2019]. This approach is taken by International Data Corporation (IDC), one of the world's largest consulting companies, a leading provider of information and consulting services, an organizer of events in the markets of information technology, telecommunications and consumer equipment [Andsbjerg R., Vesset D., 2018]. The Computing Classification System (developed by the Association for Computing Machinery, the latest version was introduced in 2012) can also be used to classify software and information systems. The system is presented as a single source of categories and concepts that reflect the current state of computing, computer science, and information and communication technologies [Computing Classification System, 2012].

The established classifications do not allow a software designed for contextual scientific knowledge processing to be assigned to one class. Such software includes various linguistic programs. A sufficient number of catalogues of such programs is found on the Internet, for example [4 Free and Open Source ..., Text Analysis, Text Mining ..., Text Mining Software ...]. They might be used for research purposes. In these directories there are no classifiers considering the main purpose of the software, types of contexts being processed, and processing functions. Therefore, they do not fulfill the task of choosing effective tools for conducting research.

Based on the analysis of software designed for contextual knowledge processing, the following general classes of tasks for contextual knowledge processing are identified: neural network; machine learning; natural language processing; information extraction; ontology; construction and analysis of trends; creation and use of thesauri; thematic classification of texts; full-text information databases; abstract databases (only metadata). These classes of tasks in the catalogue correspond to the characteristic "category (software class)". The last two classes are characterized only by full-text and abstract information databases, which have their own mechanisms for searching, selecting, and analyzing information.

The typology of contexts developed by [] allows to determine the software necessary to solve the problems of context processing. The software analysis allowed to identify the types of contexts for each application [Kononova O.V., Prokudin D.E., 2020]. Therefore, one of the essential characteristics of the software is the "view (s) of the context being processed": corpus, fragment, paragraph, sentence, concept term, thesaurus, meta description, query collection, semantic group, publications set, thematic collections of relevant paragraphs, group of keywords, subject areas.

Software classification and context typology do not adequately reflect software capabilities. Therefore, for a more complete picture of the capabilities of the software, a list of their functions is used, which is mapped to modules, services, and contexts. Functions are represented by a separate characteristic in accordance with the scientific tasks performed. Based on this, the following functions are distinguished: clustering; forecasting; contextual analysis; intelligent search; automated exchange of data (metadata); visualization; storage.

These three main characteristics are the integral basis for the rational choice of specific software to be effective for research purposes. 


\section{HUMAN-READABLE AND MACHINE-READABLE CATALOGUE REPRESENTATION}

\subsection{Architectural Description of Information Systems}

Architectural descriptions used in the catalogue are software meta-description, architectural model of applications, and taxonomy of contexts processed by software.

The software meta-description for contextual knowledge explication and processing contains the following fields: Name, URL; Vendor, country; Properties (distribution license, the latest version year, support; Software Classes (Type); Purpose; Main functions; Additional functions; List of modules, services; Types of Contexts; Links to materials on using the software).

The selection and visualization of contexts is performed in accordance with the previously developed typology of contexts [Kononova O.V., Prokudin D.E., Yelkina E.E., 2019, Kononova O.V., Prokudin D.E., 2020]. First, the contexts the information system works with are determined. Next, the contexts are compared with typology: groups and types of contexts are distinguished.

A meta-description of the application's architectural model, which determines the corresponding functions and contexts implemented in the information systems, is presented in Table 1.

Table 1. Context model of an application architecture

\begin{tabular}{l|l|l}
\hline \multicolumn{1}{c|}{ Modules / Services } & \multicolumn{1}{c}{ Functions } & \multicolumn{1}{c}{ Contexts } \\
\hline $\begin{array}{l}\text { The list of modules or services with IS } \\
\text { functions. }\end{array}$ & Functions implemented in IS. & $\begin{array}{l}\text { The list of context types } \\
\text { based on the typology. }\end{array}$ \\
\hline
\end{tabular}

The data architecture is represented in the catalogue by two artifacts: a list of entities and ERD (using Crow's Foot notation [Moss K., 2012]).

The list of entities is determined by the following criteria: types of context the system in question works with (in accordance with the typology of contexts) or other units of analysis; the results that the system provides after analysis or a form of visualization; the place for data storage. ERD describes the relations between the entities selected. This description provides information about the software for catalogue users in the most optimal variant.

\subsection{Catalogue Submission in Machine-Readable Form}

The information analysis of the software for contextual knowledge demonstrates that basically catalogue directories are static lists or tables in which the software is grouped by a certain attribute or presented in an unstructured form with a brief description of the features and links to relevant sites on the Internet.

Based on generally accepted approaches for software description in a catalogue, it is proposed to use the Dublin Core metadata presentation specification [Brisebois R. et al, 2017, González R. and Van Der Meer K., 2004]. Here, the main characteristics presented in the software catalogue collaterals are described by the relevant elements of the Dublin Core Metadata Element Set (DCMES) [DCMI Metadata Terms]. Therefore, a combination of element values s fully describes the software presented in the catalogue, which corresponds to the general approach of entity description with this specification [Fedotov A.M., Leonova Y.V., 2018, Noor S. et al, 2018]. Within the framework of this approach various text objects are described: articles, books, library catalogue cards, archival materials, and semantic models [SUNScholar / Metadata / By Function]. When developing a metadata presentation scheme for software description, its specification is not considered, which is important when choosing software applications (for example, types of processed contextual knowledge) [Brisebois R. et al, 2017, González R. and Van Der Meer K., 2004]. Allowing for software description specifics, the qualifiers were used to refine the characteristics values in addition to the main metadata elements. These qualifiers form the second level of metadata and refine the values of [DCMI Qualifiers] elements.

The catalogue in machine-readable form can be implemented as an information system with free Internet access. This catalogue presentation is convenient for users and for automated identification by search engines and aggregation into other information systems. To implement the catalogue, DSpace software platform (https://duraspace.org/dspace/) was chosen as the most common solution at present. In accordance with the Dublin Core specification, metadata presentation in DSpace [DSpace / dublin-core-types ...] and considering 
qualifier presentation, the essential software features for each catalogue element are represented by the following tags:

dc.type - categories (software classes in accordance with the developed classification)

dc.subject - keywords

dc.subject.classification - main functions (can be supplemented after the corresponding software analysis)

dc.subject.other - types of contexts processed

\section{SOFTWARE CATALOGUE: CASE STUDY}

The architecture is designed for the main group of system users - analysts, researchers, and scientists. For these users, it is important to obtain necessary information in proper volume and correct presentation. The architectural representation gives a user access to information about the system: the context the system works with, the modules implemented in the system, and also what results analysis can be performed using the system. Consider the architectural representation of T-libra and Voyant-tools information systems. Figure 1 demonstrates the context ontology for T-libra (a) and Voyant-tools (b) systems.

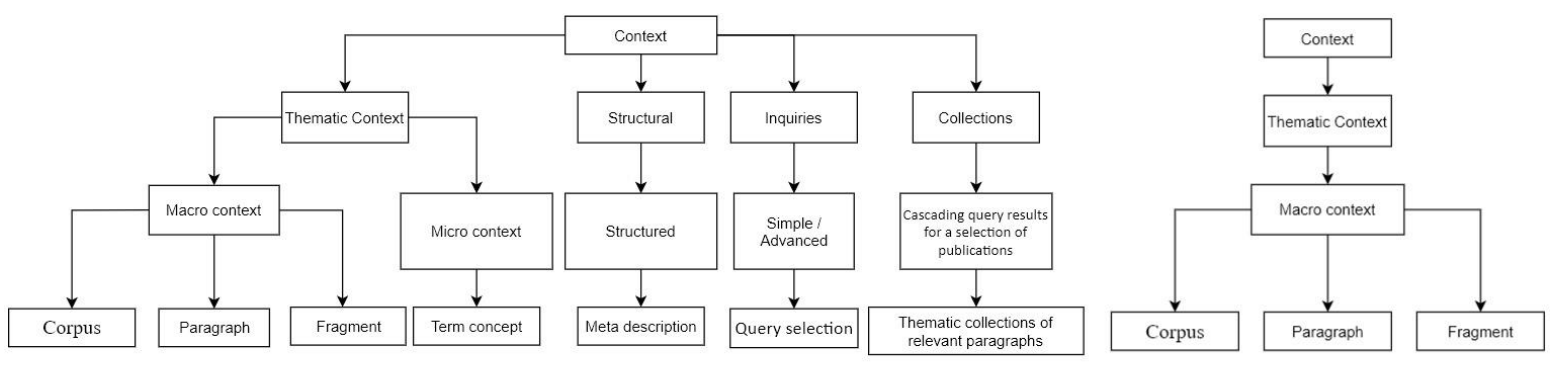

a)

b)

Figure 1. Context ontology. T-libra (a), Voyant-tools (b)

The ontology features the context the system works with. After defining the context, the users can learn more about the system functionality. Table 2 demonstrates T-libra and Voyant-tools functions and modules.

Table 2. Functions and modules of T-libra and Voyant-tools information systems

\begin{tabular}{l|l|l}
\hline \multicolumn{1}{c}{ IS Functions } & \multicolumn{1}{c}{ Module / Services } & \multicolumn{1}{c}{ T-Libra } \\
\hline Storage & $\begin{array}{l}\text { Data loading and storage data (text arrays); Depositary; } \\
\text { Catalogue }\end{array}$ & $\begin{array}{l}\text { Corpus, document, query, meta } \\
\text { description, thematic collection }\end{array}$ \\
\hline Search & $\begin{array}{l}\text { Implementation of several types of contextual search: } \\
\text { absolute and relative frequency-oriented search; paragraph- } \\
\text { oriented search (single-layer, multi-layer queries) }\end{array}$ & $\begin{array}{l}\text { term concept, paragraph, fragment, } \\
\text { query }\end{array}$ \\
\hline Analysis & $\begin{array}{l}\text { Explication of contexts by cascading query method; } \\
\text { Creation of thematic collections based on query results } \\
\text { clustering, content and expert analysis of contexts }\end{array}$ & $\begin{array}{l}\text { Thematic collection of relevant } \\
\text { paragraphs, fragment, paragraph, } \\
\text { term-concept }\end{array}$ \\
\hline Visualization & $\begin{array}{l}\text { Building of terminogramma as absolute results of } \\
\text { frequency-oriented queries; Statistics }\end{array}$ & Terminogramma (List of term) \\
\hline Search & \multicolumn{2}{c}{ Voyant-tools } \\
\hline Analysis & $\begin{array}{l}\text { Absolute and relative frequency-oriented search } \\
\text { Computer analysis to study texts on the Internet, or texts, } \\
\text { from the user's computers; functionality addition to } \\
\text { individual online collections, science magazines, blogs, or } \\
\text { websites so that other users can view texts using analytic } \\
\text { tools }\end{array}$ & $\begin{array}{l}\text { Corpus, document, paragraph, } \\
\text { fragment, compilation of publications } \\
\text { oriented search }\end{array}$ \\
\hline Visualization & $\begin{array}{l}\text { Graph construction, tables, tag clouds, bubble diagrams, } \\
\text { relationship graphs }\end{array}$ & $\begin{array}{l}\text { Corpus, document, fragment, } \\
\text { paragraph (reduced fragment), } \\
\text { compilation of publications }\end{array}$ \\
\hline
\end{tabular}


The table gives catalogue users an idea of how the basic functions (storage, search, analysis, visualization) are implemented in the system. Also, the table demonstrates the types of contexts used for a particular function.

The ERDs for T-Libra and Voyant-tools are demonstrated at Figure 2. The diagram presents the entities and their relationships. This allows a user to determine the system efficiency with a particular type of context. The diagram illustrates the relationship of this context with the analysis result or data storage location.

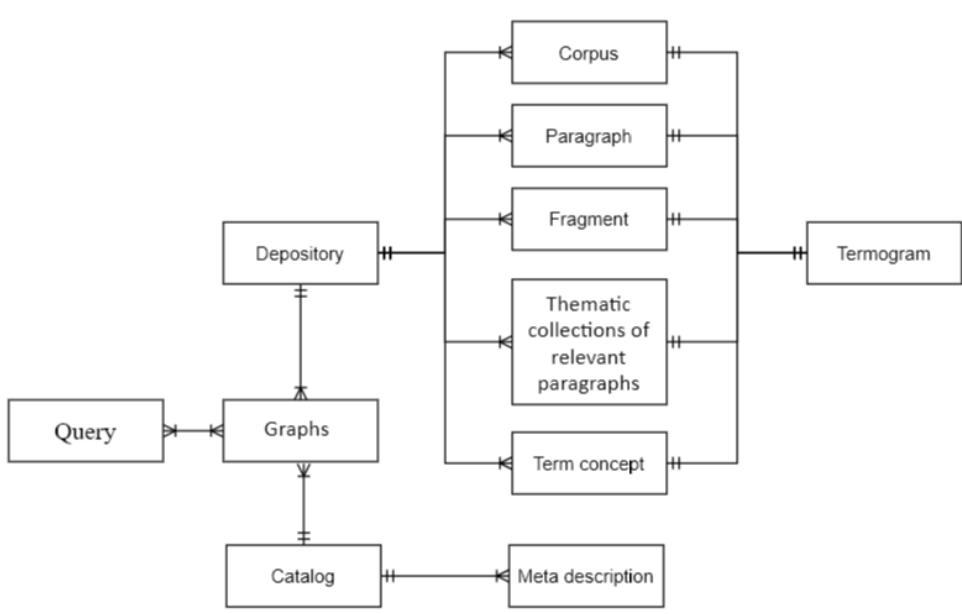

a)

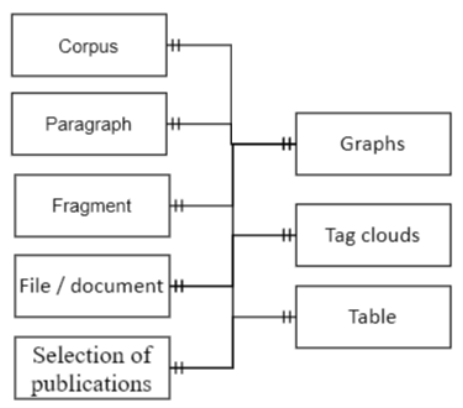

b)

Figure 2. ERD. T-libra (a), Voyant-tools (b)

Considered is a machine-readable part of the catalogue. Tables 3 and 4 provide a machine-readable description of the T-libra and Voyant-tools information systems.

Table 3. Machine-readable description of T-Libra

\begin{tabular}{|c|c|}
\hline dc.title & T-Libra \\
\hline dc.creator & LLC "Constant", Russia \\
\hline dc.subject & Collection of documents; Text Corpus; Frequency query; Contextual search; Trend; Context \\
\hline dc.subject.classification & Contextual analysis; Storage; Search \\
\hline dc.subject.other & $\begin{array}{l}\text { Case, Document, Paragraph, Fragment, Term concept, Query, Thematic collection of relevant } \\
\text { paragraphs (result of a search query followed by expert selection), Meta description }\end{array}$ \\
\hline dc.description & $\begin{array}{l}\text { information system for creating multifunctional electronic libraries with flexible thematic } \\
\text { full-text search, multimodal expansion and metadata conversion from electronic catalogues }\end{array}$ \\
\hline dc.publisher & LLC "Constant" \\
\hline \multicolumn{2}{|l|}{ dc.contributor } \\
\hline dc.date.issued & 2017 \\
\hline dc.type & Full-text information databases; Natural Language Processing; Information Extraction \\
\hline dc.format.mimetype & trtf; pdf \\
\hline dc.identifier.uri & http://www.softconst.ru/ \\
\hline dc.source.uri & http://www.softconst.ru/tlibra/ \\
\hline dc.language & Multilanguage \\
\hline dc.coverage & Web Oriented Application (Web Interface) \\
\hline dc.rights & Commercial license \\
\hline $\begin{array}{l}\text { dc.relation. } \\
\text { isreferencedby }\end{array}$ & $\begin{array}{l}\text { Kononova O., Lyapin S. (2016) Using the Contextual Search for the Organization Scientific } \\
\text { Research Activities. In: Chugunov A., Bolgov R., Kabanov Y., Kampis G., Wimmer M. (eds) } \\
\text { Digital Transformation and Global Society. DTGS 2016. Communications in Computer and } \\
\text { Information Science, vol 674, pp. 392-399. Springer, Cham. DOI: 10.1007/978-3-319-49700- } \\
6 \text { 6_38 }\end{array}$ \\
\hline dc.coverage & all \\
\hline
\end{tabular}




\begin{tabular}{|c|c|}
\hline dc.rights.license & $\begin{array}{l}\text { LLC "Constant" } \\
\text { Table 4. Machine-readable description of Voyant-tools information system. }\end{array}$ \\
\hline dc.title & Voyant-tools \\
\hline dc.creator & Stéfan Sinclair, McGill University; Geoffrey Rockwell, University of Alberta \\
\hline dc.subject & $\begin{array}{l}\text { Collection of documents; Text Corpus; Frequency query; Contextual search; Trend; } \\
\text { Explication of contextual knowledge; Context }\end{array}$ \\
\hline dc.subject.classification & $\begin{array}{l}\text { Processing of individual documents; Processing by a collection of documents (corpus of texts); } \\
\text { Classification; Clustering analysis of web pages; Frequency analysis; Contextual analysis; } \\
\text { Building trends; Visualization of analysis results }\end{array}$ \\
\hline dc.subject.other & Corpus, Document, Paragraph, Fragment, Term Concept, Thesaurus \\
\hline dc.description & $\begin{array}{l}\text { Voyant-tools is a web-based text reading and analysis environment to facilitate the work with } \\
\text { a text or collection of texts in a variety of formats, including plain text, HTML, XML, PDF, } \\
\text { RTF, and MS Word. } \\
\text { There is a local solution in the form of an application on JETTY }\end{array}$ \\
\hline dc.publisher & Voyant-tools \\
\hline dc.contributor & Andrew MacDonald; Cyril Briquet; Lisa Goddard; Mark Turcato \\
\hline dc.date.issued & 2018 \\
\hline dc.type & Natural Language Processing; Information Extraction \\
\hline dc.format.mimetype & txt; rtf; doc; docx; pdf; zip; html; xml \\
\hline dc.identifier.uri & https://voyant-tools.org \\
\hline dc.source.uri & http://voyeurtools.org/voyant-server/ \\
\hline dc.language & Multilanguage \\
\hline dc.coverage & Web Application, Mac, Windows, JETTY server, Voyant server \\
\hline dc.rights & Free Software \\
\hline $\begin{array}{l}\text { dc.relation. } \\
\text { isreferencedby }\end{array}$ & $\begin{array}{l}\text { Laurie J. Sampsel (2018) Voyant Tools, Music Reference Services Quarterly, 21:3, 153-157, } \\
\text { DOI: 10.1080/10588167.2018.1496754 }\end{array}$ \\
\hline dc.coverage & all \\
\hline dc.rights.license & Creative Commons Attribution 4.0 International (CC BY 4.0) \\
\hline
\end{tabular}

The table provides the information with convenient search engine. Thus, using the machine-readable part of the catalog, one can find the necessary information about the system and system specifics having analyzed the system architecture.

\section{CONCLUSION}

Based on the developed approach to the software systematized and structured presentation for contextual knowledge explication and processing, a software catalogue was presented in two forms - human-readable and machine-readable. The Dublin Core metadata specification was used for a structured description of the software in a machine-readable catalogue. This specification was supplemented by the DSpace software platform qualifiers. The catalogue was arranged in human readable form for further publication in a book publishing house.

The proposed approach to the software catalogue in machine-readable form is based on a Dublin Core format metadata description. Each record represents an ordered set of metadata. This provides:

- typology of contexts integrated into the catalogue developed by [Kononova O.V., Prokudin D.E., Yelkina E.E., 2019, Kononova O.V., Prokudin D.E., 2020];

-machine-readable catalogue using standard free software (for example, OJS, DSpace);

- effective search and selection of a software for research purposes in accordance with basic characteristics described in the Dublin Core tags using standard search engines;

- open access to catalogue records for both users and automated indexing;

- automated exchange using OAI-PMH protocol for aggregation of catalogue meta description to other information systems. 


\section{ACKNOWLEDGEMENT}

The reported study is funded by RFBR according to the research project «The integrated approach elaboration to the analysis of the terminological base of the developing interdisciplinary research in distributed network environment» № 18-011-00923, 2018-2020.

\section{REFERENCES}

4 Free and Open Source Text Analysis Software. https://www.softwareadvice.com/resources/easiest-to-use-free-andopen-source-text-analysis-software/

Andsbjerg R., Vesset D., 2018. IDC's Worldwide Software Taxonomy, 2018: Update. https://www.idc.com/getdoc.jsp?containerId=US44835319

Brisebois R., Abran A., Nadembega, A., 2017. A Semantic Metadata Enrichment Software Ecosystem (SMESE) Based on a Multi-Platform Metadata Model for Digital Libraries. In Journal of Software Engineering and Applications. No. 10, pp. 370-405. DOI:10.4236/jsea.2017.104022

Computing Classification System. https://dl.acm.org/ccs

DCMI Qualifiers. Dublin Core Metadata Initiative. https://www.dublincore.org/specifications/dublin-core/dcmesqualifiers

DSpace/dublin-core-types.xml at master DSpace. DSpace. GitHub. https://github.com/DSpace/ DSpace/blob/master/dspace/config/registries/dublin-core-types.xml

Fedotov A.M., Leonova Y.V., 2018. Requirements for the prototype of the information resources management system in distributed information systems for the support of scientific research. In Computational technologies. Vol. 23, № 5, pp. 82-109. DOI:10.25743/ICT.2018.23.5.008

González R., Van Der Meer K., 2004. Standard Metadata Applied to Software Retrieval. In Journal of Information Science. Vol. 30(4), pp. 300-309. DOI: 10.1177/0165551504045850

Kononova O.V., Prokudin D.E., 2020. An approach to extraction, explication and presentation of contextual knowledge in the study of developing interdisciplinary research areas. In International Journal of Open Information Technologies. Vol 8, № 1, pp. 90-101. URL: http://injoit.org/index.php/j1/article/view/882/844

Kononova O.V., Prokudin D.E., Yelkina E.E., 2019. Contextual Knowledge Extraction: Terminological Landscape of Digital Economy. In Sociology of Science and Technology. Vol. 10(3), pp. 103-120. DOI:10.24411/2079-0910-201913006

Moss K., 2012. The Entity-Relationship model // IEEE Global Engineering Education Conference, EDUCON.

Noor S., Shah L., Adil M., Gohar N., Saman G.E., Jamil S., Qayum F., 2019. Modeling and representation of built cultural heritage data using semantic web technologies and building information model. In Computational and Mathematical Organization Theory, № 25, pp. 1-24. DOI: 10.1007/s10588-018-09285-y

SUNScholar/Metadata/By Function. Libopedia. https://wiki.lib.sun.ac.za/index.php/SUNScholar/ Metadata/By_Function

Text Analysis, Text Mining, and Information Retrieval Software. https://www.kdnuggets.com/software/text.html

Text Mining Software. https://www.capterra.com/text-mining-software

Text mining, text analytics \& content analysis with free open source software. https://www.opensemanticsearch.org/doc/analytics/textmining

Woodward A., Anderson R., Biscotti F., Contu R., Gupta N., Hunter E., Hare J., Bhullar B., Dayley A., Roth C., Swinehart H., Dsilva V., Wurster L., Poulter J., Palanca T., Deshpande S., Pang C., Abbabatulla, B., Warrilow M., Dharmasthira Y., Kostoulas J., 2019. Market Definitions and Methodology: Software. Gartner, Stamford. https://www.gartner.com/en/documents/3906823/market-definitions-and-methodology-software 